\title{
Development of Numerical Methods for Simulation of Rapid Multi-Phase Interactions
}

\author{
Yanhua YANG ${ }^{\dagger}$, Hirotada OHASHI and Mamoru AKIYAMA \\ Department of Quantum Engineering and Systems Science, University of Tokyo*
}

(Received December 6, 1995)

\begin{abstract}
Techniques commonly used in the calculations of multi-phase flow, such as local parameter averaging and continuity phase assumptions, may result in large calculational inaccuracies for phenomena with rapid phase change. For example, with vapor explosions, a large degree of pseudo-diffusion (i.e. numerical diffusion) of components in dispersed states or with discontinuous fronts may occur, and local information, useful for modeling and evaluation of the phenomena, may be lost. Pseudo-diffusion of the components which act as hightemperature heat sources affects component distributions throughout the entire system. Numerical divergence is also often caused by fluctuation of these high-temperature components. In this paper, two treatment methods are proposed to avoid these problems. One method is a dispersed component method, which was developed for dispersed components. This method includes special modeling for some local parameters and some treatments to suppress pseudo-diffusion and numerical divergence. The other is a multi-region scheme, where a calculation domain is divided into an expanding, multi-phase mixture region and the surrounding, single-phase continuum region. The front of the mixture region is tracked using an adaptable grid to accurately follow the interface. By this method, numerical diffusion at the interface can be avoided. The effectiveness of these treatments is demonstrated by numerical simulations.
\end{abstract}

KEYWORDS: two-phase flow, thermal-hydraulic code, rapid multiphase interactions, vapor explosion, hot sources, local parameter averaging, continuity phase assumption, dispersed distribution, numerical analysis, numerical simulation, pseudo-diffusion, numerical divergence

\section{I . INTRODUCTION}

Rapid multi-phase interaction problems have recently received much attention in many scientific areas. In this paper, the vapor explosion phenomenon, which is often considered in analysis of severe accidents of nuclear power systems ${ }^{(1)(2)}$, is taken as a typical example for discussion. The vapor explosion may occur when two liquids with a large temperature difference come into contact. The high heat transfer rate caused by the direct contact gives rise to an extremely large evaporation rate over a very short time period, and thermodynamic variables change rapidly in both time and space.

Numerical simulations of such a rapid multi-phase interaction process, particularly for vapor explosions, have been widely studied in recent years. Many of the simulations were performed by using a one- or twodimensional, multi-component, thermal-hydraulic code, such as CHYMES ${ }^{(3)}$ and PM-ALPHA ${ }^{(4)}$. Based on a multi-fluid dynamic code, CHAMPAGNE ${ }^{(5)}$, the authors

\footnotetext{
${ }^{*}$ Hongo, Bunkyo-ku, Tokyo 113.

${ }^{\dagger}$ Corresponding author, Tel. +81-29-282-5871,

Fax. +81-29-282-5570,

E-mail: yang@sun2sarl.tokai.jaeri.go.jp

Present address: Tokai Research Establishment, Japan

Atomic Energy Research Institute, Tokai-mura, Naka-gun, Ibaraki-ken 319-11.
}

also have been developing a two-dimensional vapor explosion simulation code, CHAMP/VE, for this special application $^{(6)}$. However, due to the complicated physical mechanisms involved and the violent parameter variations in the process, calculations are limited in scope to certain phenomena, such as coarse mixing and fragmentation processes in the vapor explosion.

The existence of rapid evaporation demands treatments for such a kind of multi-phase problem be very much different from those for general multi-phase problems. In order to obtain a reasonable and convergent result, not only a series of special modeling but also some special numerical treatments are required. Moreover, some basic treatments for the governing equations, such as parameter averaging and phase continuity assumption, which are commonly used in analysis of multiphase flow, can cause a large degree of numerical diffusion and instability due to the violent parameter changes. In addition, some computational treatments, such as the methods for solving volume fraction and pressure, could affect the results very sensitively and often result in numerical divergence.

Therefore, in this paper, some problems with the application of some general treatments for multi-phase simulation in the calculation of the phenomena with rapid phase exchange are discussed, and the novel methods used in CHAMP/VE simulations for the vapor explosion 
phenomena are introduced.

First of all some major treatments for multi-phase simulations are reviewed. To mathematically describe a multi-phase system, there are two main treatments. The first is parameter averaging and the second is the phase continuity assumption $^{(7)}$. By parameter averaging, local parameters are defined as the average values in a not very small volume so that it is not necessary to know the exact locations of each component and their interfaces. Instead of this, the volume fraction parameter is used to represent the relative quantity and position of each component. The phase continuity assumption is based on this treatment. It is assumed that in the system, each component is continuously distributed, so that conservation equations similar to those used for a continuum can be applied to every component in the multi-phase system, while the interphase interactions are considered to be the outer sources in these equations.

The phase continuity assumption can cause pseudodiffusion. Within a continuum there occurs internal physical diffusion, such as thermal or viscous diffusion. However, if the component is originally discretely distributed in system, the continuity treatment would result in pseudo-diffusion within the same component from one site to another which may not be relevant to real situation. This problem becomes significant when the heat transfer of these originally discrete components plays a dominate role in the phase distributions in the system. For example, the component of high-temperature melt droplet in the system with vapor explosion takes such a position. Using the continuity treatment for this component would spread melt drops over the entire system. Although the assumed quantity would be less in the region where the high-temperature component does not exist in the real situation, it may still be enough to cause a considerable quantity of evaporation in the region, which is called pseudo-evaporation.

Another problem to be noted is that some important local parameter variations may be neglected by the process of averaging. For instance, a common assumption is that the averaged local pressure is the same for any component. However, if vapor bubbles exist and are growing, the pressure inside the bubble could be different from that at the outer side. This difference becomes large when evaporation is violent. Moreover, for vapor film boiling around a high-temperature particle, which is a typical heat transfer configuration in the coarse mixing stage of a vapor explosion, the flow within the film is an important factor in heat transfer and drag forces between adjacent components. This inner flow, however, is neglected by the averaging process.

Component classification in the system for the concerned problem is next discussed. In many of previous simulations, all vapor elements (which are usually in the states of film, bubble or continuum) were not distinguished and were combined into a single component. In practice, however, the characteristics of the vapor film located around the high-temperature component are very different from those of free bubbles. First, the mean velocity of the film is equal to that of the hightemperature component to which it is attached, while the velocity of bubbles is independent. Secondly, the characteristic size, the film thickness and the bubble radius, which are two important parameters for the constitutive relationships of heat transfer and drag forces, are difficult to distinguish when treating two different vapor structures as one.

Numerical divergence occurs often in simulations of the concerned problems. This is usually induced by an unstable variation of the volume fraction. With a high evaporation rate, the volume fraction of vapor can change rapidly and this potentially causes the numerical instability. However, the evaporation rate is affected by the distribution of high-temperature component. Thus, even though the high-temperature component may be present in a very small quantity, the fluctuation of its volume fraction affects the convergence of the calculation. Unfortunately, with the general computational methods, the components with small volume fractions often have a relatively large error in the values of their volume fractions.

Thus, in order to overcome the above problems, it is necessary to implement some novel improvements for this kind of calculation. For the present study, two methods directed against these problems have been developed. One is a dispersed component method (DCM) and the other is a multi-region scheme (MRS). The first method is specially for the components in dispersed states with small volume fractions. It includes some special phenomenological modeling for those real local parameters whose values could be very much different from the local averaged values, and some numerical treatments for avoiding pseudo-diffusion as well for overcoming numerical divergence. In addition, the vapor film is treated as a dispersed component independent from the free bubbles in the method. The second method is specially for the cases with a pouring inlet and single phase initial conditions, as illustrated in Fig. 1. In such a system, there are two distinct regions; a mixture region and a continuum phase region. This method is used to suppress numerical diffusion at the mixture region front. In this method, the calculation domain is divided into these two parts, and the interface of these two regions is tracked.

The above two methods have been partly discussed in a previous paper ${ }^{(8)}$. The aims of this paper are to discuss the theoretical basis of these methods, give a more overall description, including some new contents, and demonstrate the effectiveness of these two proposed methods. 


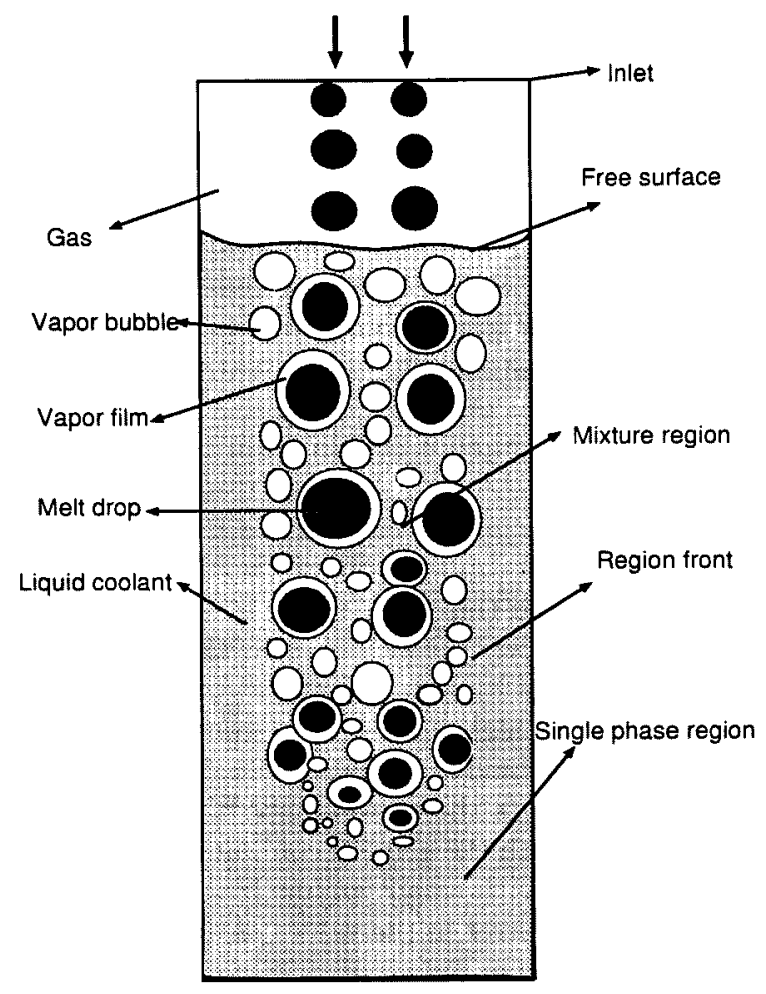

Fig. 1 A sketch for the coarse mixing stage in a vapor explosion process

\section{Description AND Approaches}

1. Dispersed Component Method (DCM)

The concept of the dispersed component method ${ }^{(6)}$ includes two parts: (1) a simplifying assumption for the diffusion terms in the governing equations, and (2) a pressure iteration scheme for improving convergence. In the present study, this method is extended by including additional treatments associated with the dispersed components and including some special modeling and numerical treatments. The main parts of this method are summarized below:

(1) Neglect of the Diffusion Terms in the Governing Equations for Dispersed Components

When the characteristic size of a dispersed element is sufficiently smaller than the size of a discrete differential cell, the physical diffusion of this component between cells can be ignored. The thermal and viscous diffusions of the dispersed components with small volume fractions may be assumed to be negliable, that is, the diffusion term in governing equations is set to be zero

$$
\frac{\partial}{\partial x_{j}}\left(\alpha_{k} \gamma_{k} \frac{\partial \phi_{k}}{\partial x_{j}}\right)=0
$$

for these components. This assumption is very effective in avoiding the pseudo-thermal diffusion for the component of the high-temperature melt drop when it is in a dispersely distributed state. Although the continuity treatment is still applied to the component, which means $\alpha_{m}>0$ at all locations, the neglect of thermal diffusion inhibits the effects of the very high melt temperature from transferring over whole system easily. Hence, if only the temperature of the melt at the place where $\alpha_{m}$ is nearly 0 is set to be the same as the initial coolant temperature, the pseudo-evaporation at these sites will be avoided to a certain degree. Pseudo-evaporation will still be presented due to numerical diffusion.

(2) Neglect of Heat Transfer in Locations where Volume Fraction is Small

Only the neglect of physical diffusion is not sufficient, because numerical diffusion can still spread to such a degree as to cause considerable pseudo-evaporation. By the first-order upwind differential scheme, the numerical diffusion by convection term is

$$
-0.5\left|u_{j}\right| \Delta x_{j} \frac{\partial^{2} \phi}{\partial x_{j}^{2}}+O\left(\delta x_{j}^{2}\right) .
$$

However, it is difficult to estimate the quantity of mass transported by numerical diffusion. But if in some place the volume fraction is very small, it may be reasonably assumed that this small mass quantity is caused by numerical diffusion. Thus, when the volume fraction satisfies the condition $\alpha_{m}<\alpha_{m i n}$, the heat transfer at this location is not calculated in the method herein. $\alpha_{\min }$ is a given constant and is taken to be a value of $1 \%$ of the inlet volume fraction of melt drops in the present calculation.

(3) Modeling for Real Local Parameters in the Vapor Film

Analysis of the vapor film in the coarse mixing stage of a vapor explosion is a typical example for explaining the difference between the real and averaged local parameters. Figure 2 illustrates the configuration of the film in a coarse mixing system. Vapor film is formed around a high-temperature melt drop and moves with the melt drop.

Since the vapor film is attached to the melt drop, it has the same coherent mean velocity as that of the melt drop. Namely, at any given location $\boldsymbol{r}$ and instant of time $t$, there is

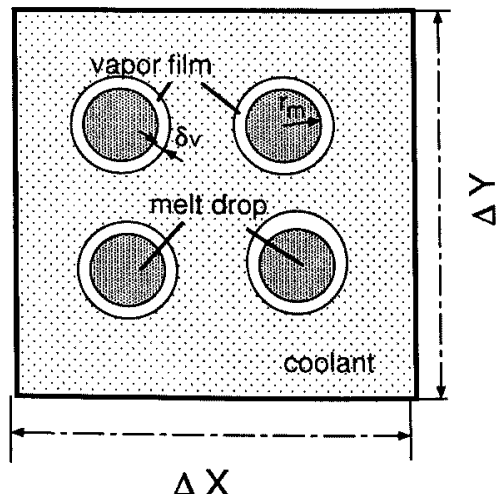

Fig. 2 The melt drops and the attached vapor films in a cell 


$$
\overline{\boldsymbol{v}}_{v}(t, \boldsymbol{r})=\overline{\boldsymbol{v}}_{m}(t, \boldsymbol{r})
$$

This mean velocity, $\overline{\boldsymbol{v}}_{\boldsymbol{v}}$, however, has almost no effect on the heat transfer within the film, while the inner local velocity, $\boldsymbol{v}_{v}(t, r, \theta(\boldsymbol{r}))$, where $\theta$ denotes the circumferential angle inside vapor film, plays a more important role in the thermal and momentum exchange around the melt. Moreover, the growth rate of the film depends on the inner-to-outer side pressure difference, as well as the relative radial velocities inside the film. If the Rayleigh equation for the growth of a bubble can be approximately applied here, then the growth rate of the film thickness can be calculated from

$$
\left(r_{m}+\delta_{v}\right) \rho_{l} \frac{\mathrm{d}^{2} \delta_{v}}{\mathrm{~d} t^{2}}+\frac{3}{2} \rho_{l} v_{v l}^{2}=p_{v}-p_{l}
$$

where $\delta_{v}$ is the thickness of the vapor film and $v_{v l}$ the relative radial velocity of the coolant-film interface. The pressures $p_{v}$ and $p_{l}$ are in the film and in the coolant, respectively. If the film is thin, the pressure in the coolant can be assumed to be the same as the average value, while the inner pressure is determined from the density and the temperature of vapor through the equation of state

$$
p_{v}=p_{v}\left(\rho_{v}, T_{v}\right)
$$

Vapor density depends on the vapor generation rate and the growth of the film thickness. From the mass balance for the vapor film, the following relationship is obtained

$$
\frac{1}{3}\left[\frac{\left(r_{m}+\delta_{v}\right)^{3}-r_{m}^{3}}{\left(r_{m}+\delta_{v}\right)^{2}}\right] \frac{\mathrm{d} \rho_{v}}{\mathrm{~d} t}+\rho_{v} \frac{\mathrm{d} \delta_{v}}{\mathrm{~d} t}=\Gamma_{v l}^{s}
$$

where $\Gamma_{v l}^{s}$ is the vapor generation flux at the film-coolant interface. If evaporation is very rapid and the growth of the film thickness is not fast enough to balance the increase of the local vapor density, the vapor density inside the film could become much higher than the value given from the corresponding averaged pressure, i.e.,

$$
\rho_{v}\left(p_{v}, T_{v}\right) \gg \rho_{v}\left(p_{l}, T_{v}\right) .
$$

As a result, the pressure in the film can be locally much larger than the averaged value. On the other hand, the volume fraction of vapor can be found from the mass equation of vapor film under the continuity assumption

$$
\frac{\partial \alpha_{v} \rho_{v}}{\partial t}+\frac{\partial \alpha_{v} \rho_{v} v_{v j}}{\partial x_{j}}=\Gamma_{v l}^{V}
$$

where $\Gamma_{v l}^{V}$ denotes the volumetric vapor generation rate. Hence, under the same vapor generation rate, the void fraction obtained from averaged variables is larger than that obtained from real local values. According to the assignment of melt drops and films in an unit volume, the following relationship between the vapor film thickness and the volume fraction can be obtained

$$
\frac{\delta_{v}}{r_{m}}=\left(1+\frac{\alpha_{v}}{\alpha_{m}}\right)^{1 / 3}-1
$$

Hence, since the density would be under-estimated by the averaging treatment (see Eq.(7)), the volume fraction and then the film thickness would be over-estimated from Eqs.(8) and (9).

From the above analysis, it may be concluded that using a local parameter averaging treatment, some important parameters, such as film thickness, local pressure, density and inner flow of the film, could be very much different from the real local values or may even be neglected. However, many constitutive relationships, such as for the vapor generation rate, the heat transfer within the film, and the drag force acting on the hightemperature drop, as well as the triggering of the followup fragmentation in a vapor explosion, are all greatly dependent on some of these parameters. Moreover, the averaging treatment also directly affects the result of volume fraction (from Eq.(8)).

Therefore, with the viewpoint that a fine calculation for the local variables is unfeasible over a large calculation gird, it becomes necessary to establish some sufficiently reasonable local phenomenological models, such as Eqs.(4) through (6), so as to obtain some important local parameters. In addition, it is also necessary to establish appropriate modeling for the local constitutive relationships for the source terms in the governing equations, which, however, will not be discussed in this paper.

(4) Classification of Vapor Film and Vapor Bubble into Independent Components

As mentioned above, vapor films and vapor bubbles (which includes the case when bubbles merge together into a continuous fluid) have different characteristics. Thus it is desirable to distinguish them as two different components. In CHAMP/VE, the vapor film is regarded as a dispersed component since it always attaches to the dispersed melt drop, while the bubble component is treated as a continuous one because it includes all vapor in any form other than that in a vapor film and its volume fraction can range from 0 to 1 . Bubbles are produced from the breakup or the ripping of the vapor film. In the present model, it is assumed that when the film thickness grows to a certain degree, the vapor bubble can be ripped from the surface of the film. The generation rate of bubbles is equal to the generation rate of the related vapor film. Both the ripping size of the film and the diameter of the bubble are specified by input. However, these values do not yet have a theoretical basis and are expected to be improved further.

(5) Improvement on the Calculation Scheme for Volume Fractions

In governing equations for multi-phase flow, the mass conservation equation for component $k$ is:

$$
\frac{\partial}{\partial t}\left(\alpha_{k} \rho_{k}\right)+\frac{\partial}{\partial x_{j}}\left(\alpha_{k} \rho_{k} u_{k j}\right)=\Gamma_{k},
$$


where $\Gamma_{k}$ is the mass source, and the unity condition of volume fraction

$$
\sum_{k} \alpha_{k}=1
$$

are directly related with the volume fractions. Hence, the volume fractions are obtained from iterative solutions of the above equations. Although the number of the equations is one more than the number of the volume fractions, since the pressure equation is derived from a combination of the mass and momentum equation, the total equations for all dependent variables are closed. There are several kinds of calculation schemes for solving volume fractions. One of the schemes is first to solve the volume fraction of each component from the mass conservation equation of itself respectively, and then to modify them by

$$
\alpha_{k}=\frac{\alpha_{k}^{\prime}}{\sum_{k^{\prime}} \alpha_{k^{\prime}}^{\prime}},
$$

which satisfies the unity condition of volume fraction (Eq.(11)). $\alpha_{k}^{\prime}$ denotes the result from the mass conservation equation.

Another scheme is to solve the volume fraction for only one of the components from the unity condition. If this component is denoted by $j$, then,

$$
\alpha_{j}=1-\sum_{k \neq j} \alpha_{k}^{\prime} \text {. }
$$

The volume fractions of other components are calculated from their mass conservation equations.

The first kind of scheme would lead to a larger error for the component with a small volume fraction. For example, a typical value of the inlet volume fraction of high-temperature melt is 0.005 (in MIXA06 data ${ }^{(8)}$ ). The error on other components would be added to this component. As a result, the vapor generation rate might fluctuate largely in different iterative procedures, which would cause instability to the equation system and lead to numerical divergence. In the second scheme, the component calculated from Eq.(13) should be the one with the largest volume fraction. This is because all errors on other components are added on this component. However, in the concerned problem, the volume fraction of any component has a large variation with time and space, i.e., there is no component which can always take the largest quantity. If using a condition such as

$$
\alpha_{j}^{\prime}=\max \left\{\alpha_{k}^{\prime}\right\}_{j}
$$

for component $j$ in Eq.(13), numerical instability may easily result, since the volume fraction of component $j$ is constantly changing.

In the present calculation, a modification from the first scheme is used

$$
\alpha_{k}=\frac{\alpha_{k}^{\prime}}{\sum_{k^{\prime} \neq q} \alpha_{k^{\prime}}^{\prime} /\left(1-\sum_{q}^{\text {high-temp. }} \alpha_{q}^{\prime}\right)} \quad k \neq q,
$$

where $q$ denotes the high-temperature components. When iteration is converged, $\alpha_{k}=\alpha_{k}^{\prime}$. In this scheme, the high-temperature components are not modified, so that the errors on other components cannot be added to the high-temperature components. By this method, the divergence can be well avoided.

(6) Improvement on Pressure Calculation

In the CHAMP/VE code, the algorithm used is the IPSA method ${ }^{(10)}$, which is based on the SIMPLE method $^{(11)}$ for single phase flow. In this method, the pressure is calculated from a disguised discrete Poisson equation. In multi-phase flow, the discrete equation can be expressed by

$$
\sum_{k} \nabla_{d}^{2} \alpha_{k} \delta p=\frac{1}{\Delta t} \sum_{k}\left[\frac{\partial \alpha_{k} \rho_{k}}{\partial t}+\nabla_{d} \cdot \alpha_{k} \rho_{k} u_{k}^{*}-\Gamma_{k}\right]
$$

where $\delta p$ is the pressure correction which approaches zero as the result converges, and $\boldsymbol{u}^{*}$ is the approximate velocity solution of the momentum equation, and $\nabla_{d}$ denotes the discrete gradient operator. The above equation is based on the sum of all components. However, it still holds if based on a single component. The solution of this equation is very sensitive to the convergence. It should be noted that the right hand side of this equation has the same form as the mass conservation equation. When the mass exchange is large, the right hand side may change significantly before the result tends to convergence, especially for components with larger volume fraction change. To avoid the divergence, it is assumed herein that the sum of the components in Eq.(16) does not include dispersed components, e.g. vapor film and melt drop. This is because the vapor film usually has a larger change between iteration, while the melt has a volume fraction too small to affect the pressure field.

Hence, the dispersed component method includes many of the treatments associated with dispersed components, especially for the high-temperature component and the vapor film, for the purpose of solving some problems with pseudo-diffusion, numerical divergence and other numerically-induced phenomena.

\section{Multi-region Scheme (MRS)}

This method suits the flow systems where two distinct regions exist: a multi-component mixture region, and a region containing only the continuity medium (It can be either the single coolant component or including also bubble component), and an explicit mass front inbetween. The pouring process, as shown in Fig. 1, is of such a structure of distribution. In this kind of process, the mixture region is developed and expanding, and the volume fractions at the front of this region are discontinuous. The application of the Eulerian method to this front will produce a large numerical diffusion and smear the front. Pseudo-evaporation, therefore, will be produced downstream of the front or an underestimated evaporation rate may result upstream of the front be- 
cause of the reduction of the mass quantity of the melt due to the diffusion. Moreover, the diffusion of the velocity can cause a mass accumulation near the front, the influence of which will be discussed in the next section.

The multi-region scheme was developed to suppress numerical diffusion at the mass front. This method includes two aspects:

\section{(1) Two Calculation Regions}

The calculation system is divided into a continuum region and a mixture region. Therefore, it is not necessary to assume small values for the volume fractions of dispersed components and calculate them in the continuum region, as was necessary in unity region system due to the phase continuity assumption. Thus, numerical diffusion of these components will not arise in the continuum region any longer. In the mixture region, the dispersed component model is applied for dispersed components, while the continuum components are calculated over the whole system. Inside both regions, the spatial differential technique is based on the Eulerian method with staggered grids.

The scheme to avoid interface smearing in the cells at the mass front is to make the front coincide with the cell sides at any time step. To achieve this, two kinds of grids are defined. One is the fixed grid which is the initially defined grid and the other is the surface grid which is designed to be adaptive with the front. Figure 3 shows these two kinds of grids and the developing scheme of the surface grid. The components of the continuous phases are calculated over entire system on the fixed grid. The

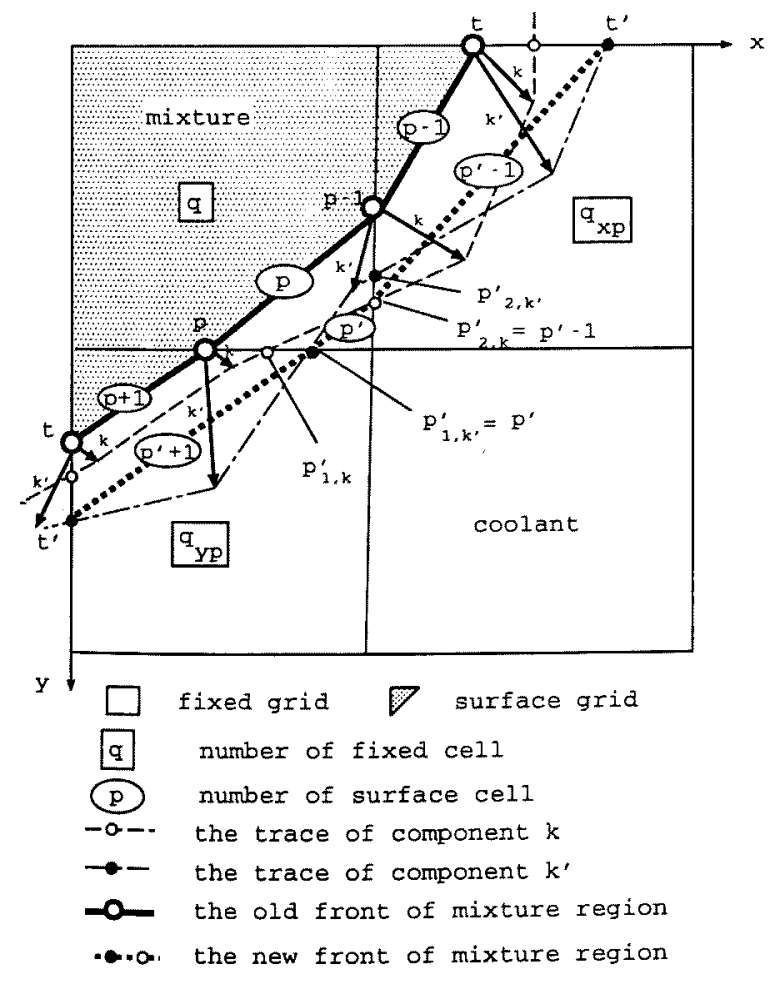

Fig. 3 The tracking scheme of the surface cells dispersed components are calculated only on the fixed grid inside the mixture region and on the surface grid at the region front.

(2) Mixture Region Front Tracking Technique

The locations and sizes of the surface cells are determined by the motion of the dispersed components on or near the interface in the mixture region. A technique has been developed to track the surface cells, which is stated below.

Assume that at any time step, the locations and the sizes of the surface cells are known. As seen in Fig. 3 , there are two points on each surface cell intersecting with the fixed grid. Suppose that all dispersed components in the mixture region consist of particles, and their velocities at these intersecting points are known at the end of previous time step. By the end of current time step, these particles will reach new locations, which are determined from $\mathrm{d} \boldsymbol{r}_{k} / \mathrm{d} t=\boldsymbol{v}_{k}$. However, the new locations of different components may be different, some of which travel far from the old mixture region, but some of which may return into the mixture region. Moreover, some particles located inside the old mixture region may travel farther than the particles on the surface at the new time step. For these reasons, it is necessary to check all dispersed components at and near the old surface front so as to judge the farthest locations which the particles reach. The new surface front is the outline of these farthest locations. Based on this consideration, the region front tracking process is performed in steps as follows:

(a) For a dispersed component $k$ on a surface cell $p$, which is located on a fixed cell $q$ and has two intersecting points numbered $p$ and $p-1$ with the cell $q$, estimate the new locations of the particles on the intersecting points from

$$
r_{p, k}^{\prime t^{\prime}}=r_{p}^{t}(q)+v_{p, k} \Delta t
$$

where $r$ denotes the location, $t$ and $t^{\prime}$ denote the old and the new instants of time and $\Delta t$ is the time step. Do the calculations for all surface cells.

(b) Connect each two adjacent new locations with a straight line and find the new intersecting points, $p_{n, k}^{\prime}$, with the fixed grid and their locations $r_{p_{n}^{\prime}, k}^{t^{\prime}}$, where $n$ expresses that there may exist more than one point in each straight line.

(c) Find the new related locations on the fixed cells for these new intersecting points: $q^{\prime}\left(p_{n, k}^{\prime}\right)$, which may be different from the original $q(p)$, such as the point $p_{2, k}^{\prime}$ which is related with $q_{x p}$ in Fig. 3 .

(d) Repeat (a) to (c) for all dispersed components $k$ to find their $\boldsymbol{r}_{p, k}^{\prime t^{\prime}}, p_{n, k}^{\prime}, \boldsymbol{r}_{p_{n, k}^{\prime}}^{\prime t^{\prime}}$ and $q^{\prime}\left(p_{n, k}^{\prime}\right)$.

(e) Check each fixed cell, $q^{\prime}$, near or on the surface to find the farthest location from

$$
\boldsymbol{r}^{t^{\prime}}\left(q^{\prime}\right)=\max \left\{\boldsymbol{r}_{p_{n, k}^{\prime}}^{t^{\prime}}\left(q^{\prime}\right), \boldsymbol{r}_{q, k}\left(q^{\prime}\right)\right\}_{k, q},
$$

where $\boldsymbol{r}_{q, k}$ denotes the new locations of the particles on the node of the fixed cell $q$ inside the mixture 
region, which is for the check of the particles which are located inside mixture region at time $t$. The results from this step are the new intersecting points at the new front of the region.

(f) Find the new velocities at these new intersecting points from a linear interpolation of velocities at the original related points.

(g) Re-number the surface cells, the intersecting points and their locations: $p^{\prime}(q), \boldsymbol{r}_{p^{\prime}}^{t^{\prime}}(q)$.

(h) Calculate other parameters based on (1) of this multi-region scheme.

(i) Estimate the new values of the velocities on intersecting points from the node value of the surface cell by intersection.

The basic idea of this method is based on the Lagrangian method for the particles at and near the surface front. However, it should be noted that the Lagrangian method is just used at each time step, which means that the particles followed are not the same ones at all time steps.

\section{Results and Discussion}

\section{General Description of the Calculation}

In order to test the effectiveness of the two methods developed in this study, several simple cases based on the melt drop pouring processes, which are commonly considered in analysis of the coarse mixing stage of a vapor explosion, were studied. The calculations were performed by the CHAMP/VE code. This code is a two-dimensional, multi-component and multi-field thermal hydraulic code. The calculation system is the same as that shown in Fig. 1, where five components are liquid coolant, melt drops, vapor film, vapor bubble and gas, were considered. Gas appears only when there is an upper gas space in the system. Descriptions of the constitutive models used in the calculations can be found in Ref.(12).

The calculation vessel is a cylinder with a radius of $200 \mathrm{~mm}$. The initial depth of coolant is $600 \mathrm{~mm}$ and the upper gas space is $450 \mathrm{~mm}$. The melt drop inlet pouring area has the radius of $70 \mathrm{~mm}$. The geometrical data are similar to the experimental data of MIXA06 ${ }^{(9)}$. The calculation region was divided into 10 nodes in the radial direction and 18 nodes in the axial direction. The inlet melt pouring area takes 4 nodes near the centerline of the container. The time step is taken from $10^{-6}$ to $10^{-3} \mathrm{~s}$, which is adjustable during the calculation. The initial conditions of the melt drops are also similar to those in MIXA06, where the melt has an inlet velocity of $5 \mathrm{~m} / \mathrm{s}$, an inlet volume fraction of 0.005 , a drop diameter of $6 \mathrm{~mm}$, the temperature of $3,600 \mathrm{~K}$, and the coolant is saturated water.

\section{Comparisons of the MRS and the URS}

The case for the validation of the MRS was calculated under the assumptions that the heat and momen- tum exchange between components could be neglected, and gravity was considered to be the only force acting on the melt drops. Under these conditions, the velocity and location of the melt drop at any instant of time can be predicted analytically. Thus, the results can easily be compared. The comparison was made between results obtained with and without the MRS. For convenience, the latter is referred to as the uniform region scheme (URS). Both of the methods are based on the DCM. The upper gas space was not included in this case. The results with two different inlet velocities are shown in Figs. 4 and 5.

Since the concern herein is the volume fraction of melt drops, the results of the volume fraction and the velocity of melt drop are only discussed. This is because the volume fraction is affected not only by the numerical diffusion of itself but also by the numerical diffusion of the velocity.

In the URS, there are two methods for treating the initial velocity of melt in the initially single-component coolant region. Since a small value for the volume fraction of melt must be always assumed here, the velocity for this small quantity should also be taken into account. It may be treated by setting the initial velocity to be zero in this region, or by giving the velocity an initial value which is equal to the inlet value and then calculating its changes in the same way as for other values of volume fraction. However, due to the numerical diffusion, these two methods will give very different results for the distributions of the velocity and the volume fraction at the sites near the front of the mixture region. The effects of these two methods are shown in Figs. 4 and 5 together, where the first method is expressed by the URS_1 and the second one is expressed by the URS_2.

Figures 4 and 5 show the profiles of velocity and volume fraction of melt drop along the centerline of the container, at the two different inlet velocities, 5.0 and 0.5 $\mathrm{m} / \mathrm{s}$, respectively. Since the only force acting on the melt is gravity, the velocity and location of the melt drops at the front of the mixture region can be estimated analytically at any given instant of time. For example, for the case with the inlet velocity of $5 \mathrm{~m} / \mathrm{s}$, at the time of 40 and $80 \mathrm{~ms}$, the expected values of velocity at the front are 5.4 and $5.8 \mathrm{~m} / \mathrm{s}$, and the expected locations are 203 and $431 \mathrm{~mm}$, respectively. The results by the MRS in Fig. 4(a) agree with the expected values very well. The same effect can be observed for the case with the inlet velocity of $0.5 \mathrm{~m} / \mathrm{s}$, as shown in Fig. $5(\mathrm{a})$. Both of the results by the URS_1 and the URS_2 show a large numerical diffusion on volume fraction and/or velocity. By the URS.1, the diffusion of the velocity at the front is large, which gives a very low value near the front and causes mass accumulation and a high volume fraction upstream of the front. The mass accumulation can reduce the loss of the volume fraction due to the numerical diffusion. However, it can become more serious with the increase of inlet 


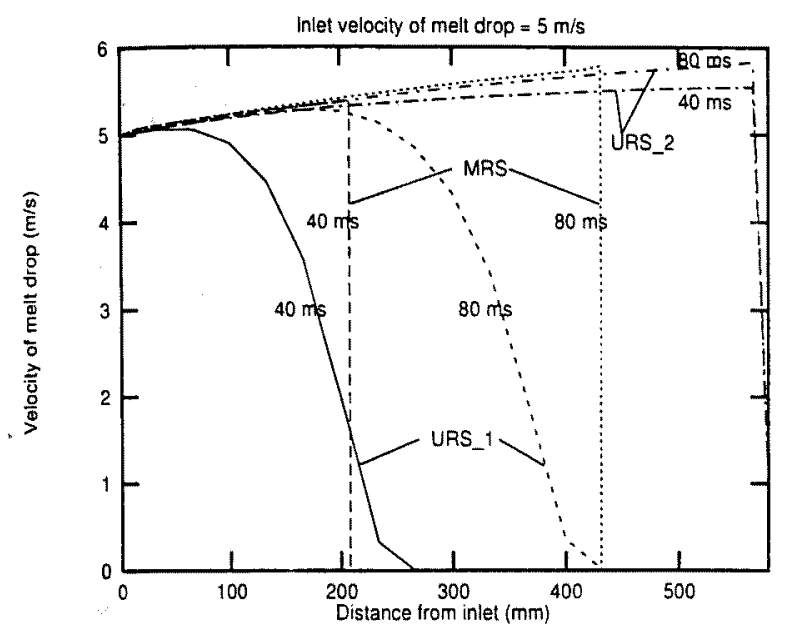

(a) Velocity of melt

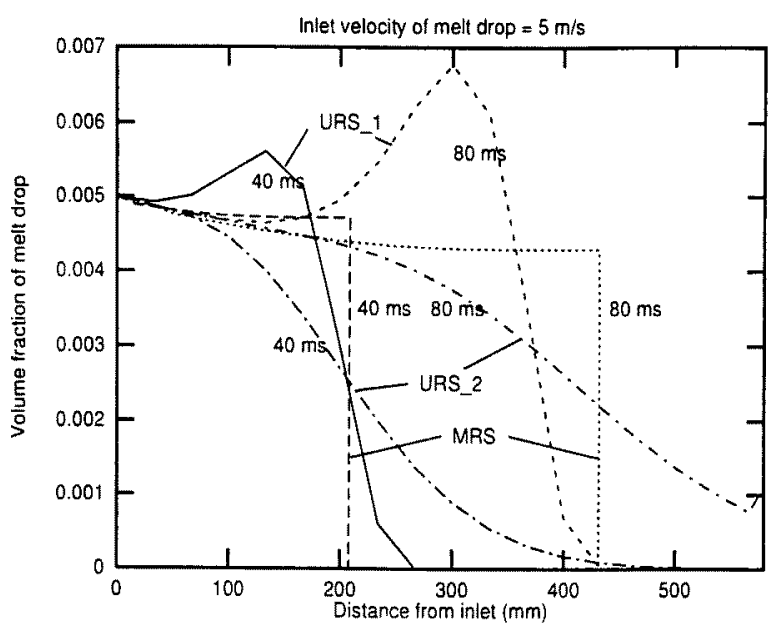

(b) Volume fraction of melt

Fig. 4 Comparisons between MRS and URS (melt inlet velocity is $5.0 \mathrm{~m} / \mathrm{s}$ )

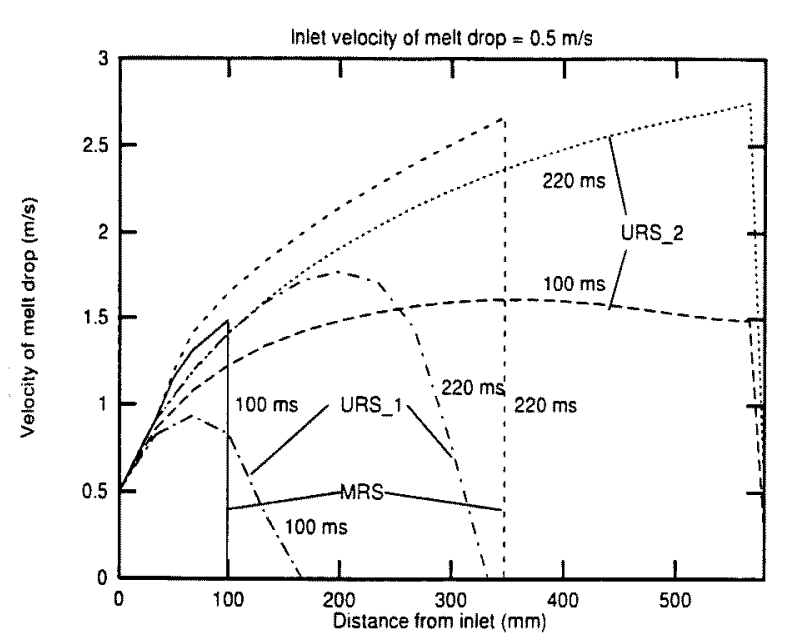

(a) Velocity of melt

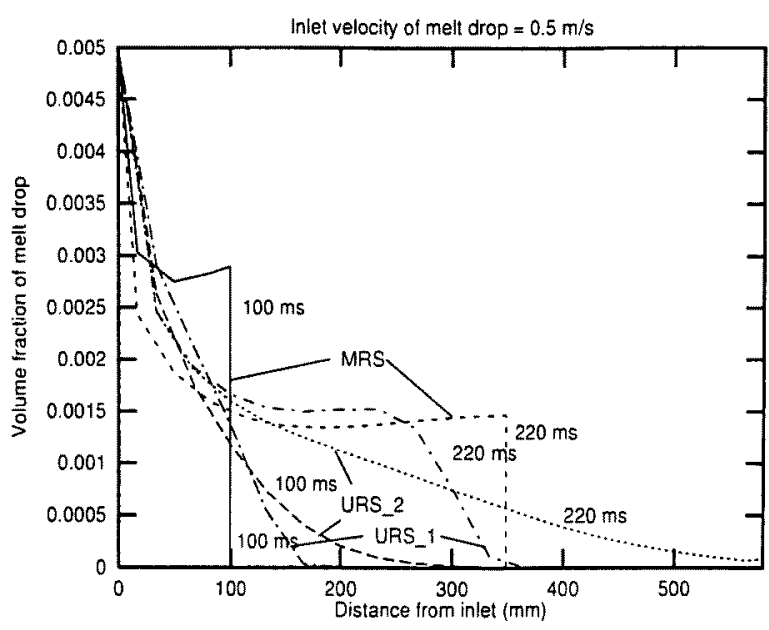

(b) Volume fraction of melt

Fig. 5 Comparisons between MRS and URS (melt inlet velocity is $0.5 \mathrm{~m} / \mathrm{s}$ )

velocities and cause another pseudo-evaporation problem at this sites (see Fig. 4(b)). By the URS_2, the diffusion on velocity can be well avoided, but the diffusion of the volume fraction becomes more serious, and the area with a relatively large value of volume fraction spreads out. Therefore, if the heat transfer is taken into account, the pseudo-evaporation will be large in the diffused area on the downstream of the front, while the evaporation on the upstream of the front will be underestimated. By the MRS, because the region front is sharp, no numerical diffusion of velocity or volume fraction is seen. The volume fraction is shown to have a value between those calculated by the URS_1 and the URS_2. Both the mass accumulation and the diffusion can be well controlled. The advantage of this method is thus evident.

\section{Comparisons of the DCM and the CFM}

The validation for the effectiveness of the DCM seems more complicated. This is because this method includes several aspects, some of which are for the purpose of avoiding divergence. Thus, it is difficult to obtain a result wholly without all of these treatments. However, some of these treatments can be still compared. One result with all treatments of DCM and another result with only some of the treatments of DCM, in which the treatment (1), (2) in Sec.II-3 were not included, were used for the comparison. The latter is referred to the continuous fluid method (CFM) here. This comparison is only used to discuss the influence by the pseudo-diffusion and the effect of the DCM on this aspect. In this calculation, heat and momentum exchanges were both taken into account. However, the drag on the melt were ignored for the purpose to avoid the larger difference on the distributions of the melt drop. The upper gas space was included in the calculation. However, to avoid the bubble going into the gas space, which can reduce the 
quantity of vapor in the coolant region and make the results difficult to analyze, the separation of the bubble from the film was not calculated in this case. Moreover, for ease in discussion, the calculation for this comparison is combined with the URS, since the diffusion on the region interface can be more distinct than in other locations. Figure 6 shows the results.

This comparison is performed with the URS.1. Figure 6 shows the temperature of melt, the volume fraction of melt and the volume fraction of vapor obtained by the DCM and the CFM. From Fig. 6(a), it is evident that, with the CFM, the pseudo-physical diffusion between discrete particles makes the temperature propagate fast over the system, which even can compensate for the reduction due to the numerical diffusion at the front. However, the temperature front moves faster than the volume fraction front, a physical impossibility, which can be observed from the comparison of Figs. 6(a) and (b). With the DCM, the temperature diffuses also at the front, which can give a low vapor generation rate at the front. Hence it can also reduce the pseudo-evaporation due to the mass accumulation at this place. Hence, even without applying the MRS, the DCM can also contribute to effectively suppress the pseudo-evaporation at the region front. While with the CFM, although the distribution of the melt does not have a large change (see Fig. $6(b)$ ) in these two methods, the amount of vapor produced in the two methods are different. In Fig. 6(c), we can find that with the increase of the time, the quantity by the pseudo-evaporation becomes large. The error can reach to $30 \%$ at the centerline. This part of the vapor is thought to be produced by the pseudo-physical diffusion. Such a large quantity of pseudo-evaporation cannot be accepted. Thus, the DCM has certain effect to suppress the pseudo-evaporation.

However, the DCM is not only effective in suppressing the pseudo-diffusion, but also effective to avoid the divergence and give a more reasonable result because of the physical modeling solved.

\section{Conclusions}

For phenomena with rapid phase interaction, especially with rapid phase change, attention should be given not only to the selection of the specific physical modeling for constitutive relationships, but also to the treatments in numerics. Some common treatments, such as parameter averaging and phase continuity assumptions, have been found to be not suitable for modeling of these phenomena, especially, when the dominant component, such as melt drops in vapor explosions, is present in small quantities. These treatments would not only be based on some unjustifiable physical assumptions, but may also lead to large numerical inaccuracies, such as pseudo-diffusion or divergence. These problems are mainly caused by the calculation of the distribution of the dominant component. Although its volume frac-

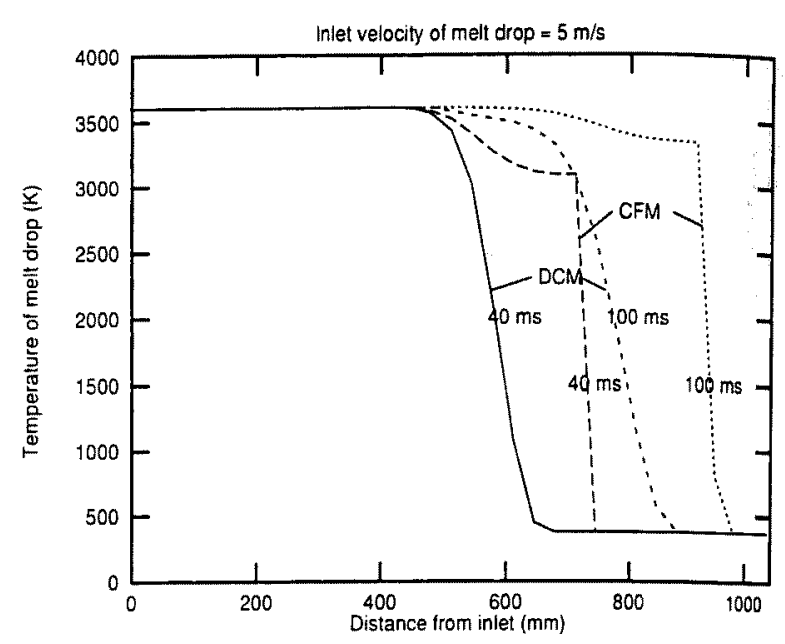

(a) Temperature of melt

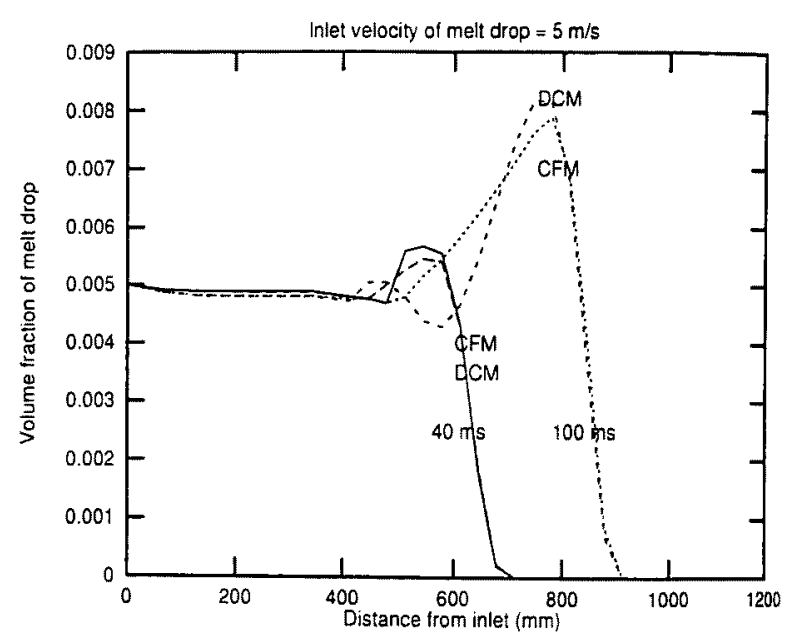

(b) Volume fraction of melt

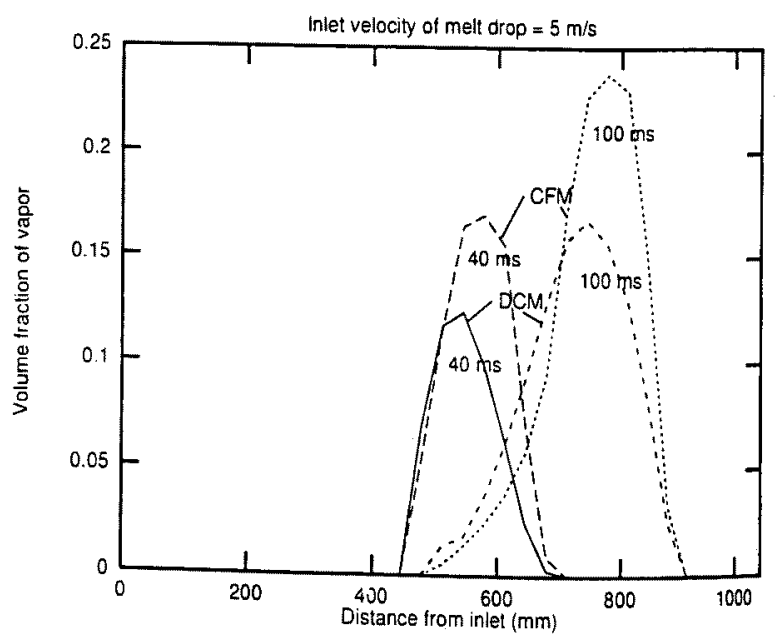

(c) Volume fraction of vapor

Fig. 6 Comparisons between DCM and CFM 
tion may be a very small value, its distribution affects all other distributions in the system. Hence, the distribution of the dominate component must be correctly calculated. The DCM and MRS methods, proposed in the present paper, can be used to partially overcome these problems. The DCM can overcome the pseudo-diffusion between particles and can also suppress the numerical divergence, especially where the cause is instability of the volume fraction. The MRS can be effectively used to suppress the numerical diffusion at the mass front of the mixture region.

\begin{tabular}{|c|c|}
\hline \multicolumn{2}{|c|}{ [NOMENCLATURE] } \\
\hline $\begin{aligned} & p: \text { Pressure } \\
& r, r: \text { Radius, location, } \\
& t: \text { Time } \\
& x_{j}, y_{j}: \text { Size of cell } \\
& \text { (Greek Symbols) }\end{aligned}$ & $\begin{array}{l}v, v: \text { Velocity } \\
S: \text { Source } \\
T: \text { Temperature }\end{array}$ \\
\hline $\begin{array}{l}\alpha: \text { Volume fraction, } \\
\Gamma: \text { Vapor generation rate, } \\
\rho: \text { Density, } \\
\text { (Subscript) }\end{array}$ & $\begin{array}{l}\gamma: \text { Diffusion coefficients } \\
\delta: \text { Thickness of vapor } \\
\phi: \text { Essential quantities }\end{array}$ \\
\hline $\begin{array}{l}l: \text { Liquid, } \\
m: \text { Melt drop, } \\
q: \text { Number of fixed cell, } \\
v: \text { Vapor film, }\end{array}$ & $\begin{array}{l}k: \text { Identifier of components } \\
p: \text { Number of surface cell } \\
s: \text { Surface } \\
V: \text { Volume }\end{array}$ \\
\hline
\end{tabular}

-References-

(1) Corradini, M.L., Kim, B.J., Oh, M.D.: Prog. Nucl. Energy, 22[1], 1-177 (1988).

(2) Corradini, M.L.: Nucl. Safety, 32[3], 337-362 (1991).

(3) Fletcher, D.F., Thyagaraja, A.: Prog. Nucl. Energy, 26, 31-61 (1991).

(4) Amarasooriya, W.H., Theofanous, T.G.: Nucl. Eng. Des., 126, 23-39 (1991).

(5) Morii, T., Ogawa, Y.: Nucl. Technol, 115, 333-341 (1996).

(6) Ohashi, H., Takano, T., Yang, Y., Akiyama, M., Morii, T.: Comput. Assisted Mech. Eng. Sci., 2, 79-86 (1995), [in Polska].

(7) Lahey, R.T.: "Boiling Heat Transfer: Modern Developments and Advances", Elsevier Sci. Publ. B.V., (1992).

(8) Yang, Y., Ohashi, H., Akiyama, M.: The Numerical Methods for the Development of the Mixture Region in the Vapor Explosion Simulations, Proc. NURETH-7, Vol.3, 1654 (1995)

(9) Fletcher, D.F., Denham, M.K.: Validation of the CHYMES mixing model, Proc. CSNI-FCI Specialists Meeting, (1993).

(10) Spalding, D.B.: Mathematical Methods in NuclearReactor Thermal Hydraulics, Paper presented at the Americal Society meeting on Nuclear-Reactor Thermal Hydraulics, Saratoga, NY, (1980).

(11) Fletcher, C.A.J.: "Computational Techniques for Fluid Dynamics", Vol.I, II, Springer-Verlag, (1991).

(12) Yang, Y., Ohashi, H., Akiyama, M.: A TwoDimensional Numerical Simulation of the Fragmentation Propagation in the Vapor Explosion, Proc. ICONE-3, Vol.1, 565 (1995). 